\title{
Treatment and secondary prophylaxis of venous thromboembolism with direct oral anticoagulants in patients with severe hereditary thrombophilia
}

\author{
Pasquale Madonna, Anna Guida, Maria Gabriella Coppola, Paolo Tirelli, Ernesto Grasso \\ U.O.C. Medicina Interna, P.O. Ospedale del Mare, ASL NA 1 Centro, Napoli, Italy
}

\begin{abstract}
Deficiency of protein C (PC), protein S (PS), antithrombin III (AT III), and homozygosity or combined heterozygosity for Factor V Leiden (FVL) and Factor II (FII) 20210A mutation represent severe hereditary thrombophilia (SHT) and are associated with a higher risk of early-onset venous thromboembolism (VTE). In literature, few papers have described the efficacy and safety of therapy with direct oral anticoagulants (DOACs) in VTE occurring in patients with SHT. In our setting, we identified 8 patients who have suffered from early-onset VTE and underwent therapy with DOACs (6 rivaroxaban, 2 apixaban). Among them, 2 AT III deficiency, 2 PC deficiency, 3 PS deficiency, 1 combined heterozygosity for FVL, and FII 20210A were detected. During the follow-up, neither recurrences of VTE nor hemorrhagic episodes were observed. This report describes the efficacy and safety of therapy with anti-Xa in the treatment and secondary prophylaxis of VTE in patients with SHT.
\end{abstract}

\section{Introduction}

Patients with inherited thrombophilias are known to be at increased risk of early-onset venous thromboembolism (VTE). The most common inherited predisposing factors to thrombosis are heterozygous Factor II 20210A mutation and heterozygous FV Leiden muta-

Correspondence: Pasquale Madonna, U.O.C. Medicina Interna, P.O. Ospedale del Mare, ASL NA 1 Centro, Napoli, Italy.

E-mail: linomadonna@libero.it

Key words: Venous thromboembolism; hereditary thrombophilia; direct oral anticoagulants.

Conflict of interests: the authors declare no potential conflict of interests.

Availability of data and materials: our data are available in the database of our Ambulatory of Thrombosis and Haemostasis.

Ethics approval and consent to participate: this work has been carried out according to the WMA declaration of Helsinki ethical principles for medical research involving human subjects.

Received for publication: 1 August 2020.

Revision received: 5 October 2020.

Accepted for publication: 6 October 2020.

This work is licensed under a Creative Commons Attribution NonCommercial 4.0 License (CC BY-NC 4.0).

${ }^{\circ}$ Copyright: the Author(s), 2021

Licensee PAGEPress, Italy

Italian Journal of Medicine 2021; 15:45-47

doi:10.4081/itjm.2021.1351 tion (FVL), which are detected in about $3-5 \%$ of the general population and increased thrombotic risk of 25 times with an annual incidence $<0.5 \%{ }^{1,2}$ Inherited deficiency of natural anticoagulants, antithrombin III (AT III), protein C (PC), protein S (PS) is rare $(<0.5 \%$ of the general population) but associated with an increased risk of thrombosis (50- or 10-fold increased risk of AT III or PC and PS deficient patients respectively) with an annual incidence $>1 \%$. Homozygous FVL and prothrombin Factor II (FII) 20210A, or combined defects, are also rare and, together with inherited deficiency of natural anticoagulants, represent the severe hereditary thrombophilia (SHT).

Vitamin K antagonists (VKA) have been considered for several decades the gold standard of VTE treatment in patients with SHT. Regardless, direct oral anticoagulants (DOACs), in the last decade, showed the same efficacy of VKA and a significative risk reduction of major, intracranial, and fatal bleeding. ${ }^{3,4}$ Their fewer drug and alimentary interactions, the predictable dose response, and no need for laboratory monitoring of the international normalized ratio resulted in better adherence to anticoagulant therapy. ${ }^{5}$ In the literature, only few case-reports have described the efficacy and safety of DOACs in treating VTE in patients with SHT. ${ }^{6}$ This is related to the rarity of congenital anomalies and consequently to the lack of significance of statistical analysis in the registration studies of DOACs. Among the latter, only RE-COVER I e II showed that the efficacy and safety of therapy with dabigatran was similar between patients with SHT and the entire population of patients. However, the small number of the first group of patients (11 with AT III deficiency and 25 with PC or PS deficiency) enabled to reach statistical signifi- 
cance. ${ }^{7}$ A recent review supports DOAC use for congenital thrombophilia, especially in the high-risk subgroups, and shows how the identification of the exact type of SHT in these studies would help assess the treatment selection. ${ }^{8}$

\section{Materials and Methods}

Between March 2014 and March 2019, among patients who have referred to our ambulatory of hemostasis and thrombosis, we have identified 8 subjects (1 M, 7 F) with a history of juvenile VTE and SHT (age at the first event 22-59 years, mean age $41 \pm 13.7$ years). All patients had a history of at least one VTE episode, deep vein thrombosis (DVT) of the lower limbs, and/or pulmonary thromboembolism (PE). The DVT diagnosis was clinically suspected and confirmed by color duplex ultrasonography, while PE was detected by computed tomography (CT). Venous thrombosis of the upper extremities and of atypical sites (spleno-mesenteric and portal thrombosis and cerebral venous thrombosis) were excluded from our setting. In all cases, VTE was treated with DOACs: 6 with rivaroxaban and 2 with apixaban (anti-Xa both). In 3 cases (Case 2, 3, and 8), patients affected by SHT who underwent longterm anticoagulant therapy were switched from VKA (after 10, 4, and 3 years treatment, respectively) to anti$\mathrm{Xa}$ at the recommended doses. On the other hand, 5 patients who were treated with DOACs for 3-6 months from the very beginning of VTE treatment, after the detection of severe thrombophilia (AT III, PC or PS deficiency, homozygous or double heterozygous FVL and FII 20210A mutation) prolonged therapy with DOACs, after giving informed consent. Among these 8 patients, we identified 2 AT III deficiencies, $2 \mathrm{PC}$ deficiencies, 3 PS deficiencies and, 1 combined heterozygosis for FVL and FII 20210A. Patients with moderate hyperhomocysteinemia or antiphospholipid syndrome were excluded, the latter, because it is not a type of hereditary thrombophilia and treatment with DOACs, is not recommended, the first because it is considered a type of mild hereditary/acquired thrombophilia. In our setting, 3 patients with transitory acquired prothrombotic conditions (case 4 and 6 estroprogestinic drugs and case 5 puerperium) underwent anticoagulation with anti-Xa for 12-24 months before stopping, while for the other 5 patients, lifelong therapy was considered. All patients gave informed consent before starting therapy with anti-Xa as described in the article. All authors confirm that the research was conducted within the guidelines under the terms of all relevant local legislation, and after Ethics Committee approval. Endpoints were assessed through outpatient follow-up; each one of the 8 patients underwent clinical, laboratoristic (e.g., coagulation parameters, d-dimer) and instrumental (e.g., color duplex ultrasonography and/or CT Scan) evaluation for thrombotic recurrence every three months and at the same time was screened for clinical, laboratoristic (e.g., hemoglobin and ferritin levels) and instrumental (endoscopy) evidence of hemorragic complications.

\section{Results}

Patients have been taking anti-Xa for a period of observation lasting overall 242 months $(30.2 \pm 16.3$ mean \pm standard deviation). During this time, no thrombotic recurrences nor hemorrhagic events were observed. This was true either for patients who had withdrawn therapy with anti-Xa after a short time (1224 months) or for those whose therapy had been continued for a longer time. No differences for sex, type of congenital anomalies, or therapy (apixaban or rivaroxaban) were observed (Table 1).

Table 1. Characteristics of patient population.

\begin{tabular}{|c|c|c|c|c|c|c|c|c|c|}
\hline Case & Patient & Sex & Age $1^{\circ}$ event & VTE & $\begin{array}{l}\text { Provoked vs } \\
\text { unprovoked }\end{array}$ & Drug & $\begin{array}{c}\text { Months of } \\
\text { therapy }\end{array}$ & $\begin{array}{c}\text { Type of } \\
\text { thrombophilia }\end{array}$ & $\begin{array}{c}\text { VTE } \\
\text { bleeding }\end{array}$ \\
\hline 1 & DO & $\mathrm{F}$ & 59 & PE & $\mathrm{U}$ & Riv. & 60 & AT III & $0 / 0$ \\
\hline 2 & $\mathrm{DA}$ & $\mathrm{F}$ & 49 & DVT & $\mathrm{U}$ & Riv. & 38 & AT III & $0 / 0$ \\
\hline 3 & $\mathrm{BP}$ & $\mathrm{F}$ & 41 & DVT rec & $\mathrm{P}$ (caval atresia) & Riv. & 40 & PS & $0 / 0$ \\
\hline 4 & MB & $\mathrm{F}$ & 22 & $\mathrm{PE}$ & $\mathrm{P}$ (estrogen) & Riv. & 18 and stop & $\mathrm{PC}$ & $0 / 0$ \\
\hline 5 & DI & $\mathrm{F}$ & 28 & $\mathrm{PE}$ & $\mathrm{P}$ (puerperium) & Apix & 12 and stop & FVL/FII +/- & $0 / 0$ \\
\hline 6 & $\mathrm{MI}$ & $\mathrm{F}$ & 36 & DVT & $\mathrm{P}$ (estrogen) & Riv. & 24 and stop & PS & $0 / 0$ \\
\hline 7 & $\mathrm{ME}$ & $\mathrm{M}$ & 34 & DVT rec & $\mathrm{U}$ & Apix & 36 & $\mathrm{PC}$ & $0 / 0$ \\
\hline 8 & IA & $\mathrm{F}$ & 59 & $\mathrm{DVT}+\mathrm{PE}$ & $\mathrm{U}$ & Riv. & 14 & PS & $0 / 0$ \\
\hline Total 8 & - & $7 \mathrm{~F} / 1 \mathrm{M}$ & $\begin{array}{c}41 \pm 13.7 \\
\text { mean } \pm \text { SD }\end{array}$ & - & - & $\mathrm{R} / \mathrm{A} 6 / 2$ & $\begin{array}{c}\text { Total } 242 \\
30.2 \pm 16.3 \\
(\text { mean } \pm \text { SD) }\end{array}$ & - & $0 / 0$ \\
\hline
\end{tabular}

VTE, venous thromboembolism; PE, pulmonary embolism; U, unprovoked; DVT, deep vein thrombosis, P, provoked; SD, standard deviation. 


\section{Discussion and Conclusions}

This report describes the efficacy and safety of the use of anti-Xa (rivaroxaban and apixaban) in treatment and secondary prophylaxis of VTE in patients with high-risk hereditary thrombophilia. During the observation, no thrombotic recurrences were observed. These data contrast with previous studies where the efficacy of therapy with DOACs in two patients with PS deficiency had not been demonstrated. ${ }^{9}$ Moreover, several authors had already suggested using DOACs to treat VTE in patients with severe PC deficiency; ${ }^{10}$ their efficacy being independent of the reduction of PC levels and lacking from the side effect of warfarininduced skin necrosis. ${ }^{11}$ In a recent 5-year study evaluating 33 patients with severe hereditary thrombophilia treated with DOACs, only 3 thrombotic recurrence cases were observed. They were all associated with lower medication adherence when patients underwent surgical procedures or experienced minor bleeding episodes. ${ }^{6}$ Moreover, in the acute phase, intensity and duration of therapy with oral anticoagulants was not different among patients with or without SHT. Therefore, the diagnostic evaluation for prolonged secondary prophylaxis could be postponed after the end of the acute phase as in 5 out of 8 patients of our setting. In fact, most of our patients discovered to be thrombophilic after they underwent therapy with anti-Xa for 3-6 months. On the other hand, in our setting, there were patients who suffered from VTE and knew to be affected from SHT; this subgroup was eligible for lifelong anticoagulant therapy and asked, after giving informed consent, to be treated with DOACs. In these cases, we used a long term therapy for the elevated thrombotic risk without a dose-reduction as suggested by registration studies, and no bleeding episodes nor thrombotic recurrences were observed. As described in a recent review, ${ }^{8}$ our small setting suggests the efficacy and safety of therapy with anti-Xa (rivaroxaban and apixaban) in the treatment and secondary prophylaxis of VTE in patients with SHT. Further more extensive multicentric studies are needed to obtain statistical significance and guidelines recommendation.

\section{References}

1. Mannucci PM, Franchini M. Classic thrombophilic gene variants. Thromb Haemost 2015;114:885-9.

2. Franchini M, Veneri D, Salvagno GL, et al. Inherited thrombophilia. Crit Rev Clin Lab Sci 2006;43:249-90.

3. Agnelli G, Buller HR, Cohen A, et al. Oral apixaban for the treatment of acute venous thromboembolism. N Engl J Med 2013;369:799-808.

4. Einstein Investigators; Bauersachs R, Berkowitz SD, et al. Oral rivaroxaban for symptomatic venous thromboembolism. N Engl J Med 2010;363:2499-510.

5. van Es N, Coppens M, Schulman S, et al. Direct oral anticoagulants compared with vitamin $\mathrm{K}$ antagonists for acute venous thromboembolism: evidence from phase 3 trials. Blood 2014;124:1968-75.

6. Undas A, Goralczyk T. Non-vitamin K antagonist oral anticoagulants in patients with severe inherited thrombophilia: a series of 33 patients. Blood Coagul Fibrinol 2017;28:438-42.

7. Schulman S, Eriksson H, Goldhaber S, et al. Influence of thrombophilia on the efficacy of dabigatran versus warfarin for the extended treatment of acute venous thromboembolism in RE-MEDYTM. Blood 2014;124: A1544.

8. Alameddinea R, Nassabeina R, Le Galb G, et al. Diagnosis and management of congenital thrombophilia in the era of direct oral anticoagulants. Thromb Res 2020;185:72-7.

9. Wypasek E, Potaczek DP, Alhenc-Gelas M, Undas A. PROS1 mutations associated with protein S deficiency in Polish patients with residual vein obstruction on rivaroxaban therapy. Thromb Res 2014;134:199-201.

10. Boey JP, Jolley A, Nicholis C, et al. Novel protein C gene mutation in a compound heterozygote resulting in cathastrophic thrombosis in early adulthood: diagnosis and long-term treatment with subcutaneous protein $\mathrm{C}$ concentrate. Br J Haematol 2016;172:811-3.

11. Hermans C, Eeckhoudt S, Lambert C. Dabigatran etexilate $(\operatorname{Pradaxa}(\mathrm{R}))$ for preventing warfarin-induced skin necrosis in a patient with severe protein $\mathrm{C}$ deficiency. Thromb Haemost 2012;107:1189-91. 